\title{
Arctigenin suppresses fibroblast activity and extracellular matrix deposition in hypertrophic scarring by reducing inflammation and oxidative stress
}

\author{
LING JIANG，YING DENG，WEI LI and YANG LU \\ Department of Plastic Surgery, Chongqing University Central Hospital, Chongqing 400000, P.R. China
}

Received February 27, 2020; Accepted August 18, 2020

DOI: $10.3892 / \mathrm{mmr} .2020 .11539$

\begin{abstract}
Hypertrophic scars (HSs) are a progressive fibroproliferation disorder caused by abnormal tissue repair after deep skin injury, and are characterized by continuous activation of fibroblasts and excessive deposition of extracellular matrix. Arctigenin (ATG), a phytomedicine derived from certain plants, displays antifibrotic effects in certain diseases, such as oral submucous fibrosis and peritoneal fibrosis. In the present study, to determine the antifibrotic potential of ATG in HS, a bleomycin (BLM)-induced skin fibrosis murine model was established. C57BL/6 mice were randomly divided into Control group, BLM group and BLM+ATG group. At 1 day post-bleomycin induction, the BLM+ATG group was intraperitoneally injected with $3 \mathrm{mg} / \mathrm{kg} /$ day ATG for 28 consecutive days. Pathological changes in the skin tissues were observed by hematoxylin and eosin staining. Collagen content was determined using a Sircol Collagen assay kit. Immunofluorescence staining was performed to detect the expression of TGF- $\beta 1$ and $\alpha$-SMA. The expression changes of various factors were detected by reverse transcription-quantitative PCR, western blotting and ELISA. Compared with the BLM group, ATG treatment significantly alleviated skin fibrosis by reducing dermal thickness, collagen content and expression levels of
\end{abstract}

Correspondence to: Dr Yang Lu, Department of Plastic Surgery, Chongqing University Central Hospital, 1 Jiankang Road, Yuzhong, Chongqing 400000, P.R. China

E-mail: yanglu1435@outlook.com

Abbreviations: HS, hypertrophic scar; ATG, arctigenin; BLM, bleomycin; $\alpha$-SMA, $\alpha$-smooth muscle actin; Colla1, collagen type I alpha 1 chain; Colla2, collagen type I alpha 2 chain; CTGF, connective tissue growth factor; PAI-1, plasminogen activator inhibitor-1; MCP-1, monocyte chemoattractant protein-1; GSH, glutathione; SOD, superoxide dismutase; MDA, malondialdehyde; Nrf2, nuclear factor erythroid-2-related factor 2; HO-1, heme oxygenase-1; Con, control; H\&E, hematoxylin and eosin; RT-qPCR, reverse transcription-quantitative PCR

Key words: arctigenin, hypertrophic scar, fibroblast, extracellular matrix, transforming growth factor $\beta 1$, inflammation, oxidative stress extracellular matrix-related genes (collagen type I $\alpha 1$ chain, collagen type I $\alpha 2$ chain, connective tissue growth factor and plasminogen activator inhibitor-1) in BLM-induced fibrotic skin. ATG also inhibited the transformation of fibroblasts into myofibroblasts in vivo and decreased the expression of TGF- $\beta 1$ in BLM-induced fibrotic skin. Furthermore, the contents of proinflammatory cytokines, including IL-1 $\beta$, IL-4, IL-6, TNF- $\alpha$ and monocyte chemoattractant protein-1, were significantly decreased in the BLM+ATG group compared with the BLM group. Redox imbalance and oxidative stress were also reversed by ATG in BLM-induced fibrotic skin, as demonstrated by the upregulation of antioxidants (glutathione and superoxide dismutase) and downregulation of oxidants (malondialdehyde) in the BLM+ATG group compared with the BLM group. Moreover, the results indicated that the antioxidant effect of ATG may occur via activation of the nuclear factor erythroid-2-related factor 2/heme oxygenase-1 signaling pathway. Collectively, the present study indicated that ATG could ameliorate skin fibrosis in a murine model of HS, which was partly mediated by reducing inflammation and oxidative stress. Therefore, ATG may serve as a therapeutic agent for HSs.

\section{Introduction}

Hypertrophic scars (HSs) are the pathological result of abnormal tissue repair after deep skin injury, with typical clinical symptoms manifesting as redness, thickening, stiffening, itching and tenderness (1). In patients with severe skin trauma or burn, the incidence of HS is relatively high $(30-72 \%)(2,3)$. When HSs occur in the face, joints, hands and other important tissues, fibrotic contracture causes functional limitations and aesthetic disfigurement, which can result in a physiological and psychological burden on patients (4). At present, there are a number of treatment strategies available for the prevention and treatment of HSs, including corticosteroids, cryotherapy, pressure therapy, laser, radiation and surgery (5), but the therapeutic effects are not satisfactory. Therefore, the development of effective therapeutic drugs is important.

HSs are a progressive fibroproliferation disorder that are characterized by continuous activation of fibroblasts and excessive extracellular matrix deposition (1). Following skin injury, fibroblasts surrounding the wound can be transformed into myofibroblasts, which synthesize and secrete extracellular 
matrix, serving a substantial role in wound contraction and physical support (6). Under normal circumstances, when wound healing is complete, myofibroblasts gradually undergo apoptosis, resulting in the restoration of the synthesis and degradation of extracellular matrix to a dynamic equilibrium state. However, under pathological conditions, due to high levels of inflammation and oxidative stress, excessive myofibroblasts cannot be removed and thus remain in a proliferative and activated state, leading to the synthesis and secretion of large amounts of extracellular matrix, ultimately generating thick and hardened scars $(1,6)$.

Arctigenin (ATG), a phenylpropanoid dizbenzylbutyrolactone lignin, is derived from certain plants, such as Arctium lappa, Torreya nucifera and Bardanae fructus (7). ATG is primarily used as a component of compound prescriptions in China and other Asian countries for the treatment of inflammatory diseases, including anemopyretic cold, cough, measles and syphilis (8). Numerous studies have demonstrated that ATG displays a variety of biological activities, including anti-inflammatory, antioxidant, and antineoplastic properties (9-12). In addition, ATG has also been reported to reduce fibrosis in various fibrotic diseases (13-17). For instance, in oral submucous fibrosis, ATG prevents arecoline-induced myofibroblast transdifferentiation and dysregulated activities potentially by downregulating the LINC00974-mediated TGF- $\beta$ /phosphorylated (p-) Smad 2 signaling pathway (13). In a peritoneal fibrosis model using an in vitro cell culture model of TGF- $\beta 1$-stimulated human peritoneal mesothelial cells, ATG suppressed TGF- $\beta 1$-induced epithelial mesenchymal transition in a dose-dependent manner, partly by enhancing the activity of the AMP-activated protein kinase/NF-kB signaling pathway and inhibiting the expression of plasminogen activator inhibitor-1 (PAI-1) (14). However, whether ATG can alleviate hypertrophic scarring is not completely understood.

\section{Materials and methods}

Animal and ethics statement. A total of 30 SPF-grade healthy female C57BL/6 mice (age, 7-8 weeks; weight, $23 \pm 3 \mathrm{~g}$ ) were purchased from Shanghai SLAC Laboratory Animal Co., Ltd. Mice were housed at $22-25^{\circ} \mathrm{C}$ with $50-60 \%$ humidity, $12-\mathrm{h}$ light/dark cycles, ad libitum access to food and water, and adaptive feeding for one week before conducting experiments. All animal experiments were approved by the Ethics Committee of Chongqing University Central Hospital (approval no. 2018-032).

Animal model. A bleomycin (BLM)-induced skin fibrosis murine model of HS was established as previously described (18). Briefly, a $3 \mathrm{~cm}^{2}$ area of hair on the back of the mice was removed using depilatory cream. BLM (Sigma-Aldrich; Merck KGaA) was dissolved in PBS (Wuhan Boster Biological Technology, Ltd.) and sterilized by filtration. BLM $(100 \mu \mathrm{l} ; 1 \mathrm{mg} / \mathrm{ml})$ was subcutaneously injected into a $1 \mathrm{~cm}^{2}$ area on the hair removal area using a 27-gauge needle. The injections were administered daily for 21 consecutive days.

Experimental design. A total of 30 C57BL/6 mice were randomly divided into three groups $(n=10$ per group): i) Control (Con); ii) BLM; and iii) BLM+ATG. HS modeling was established in the BLM and BLM+ATG groups, whereas the Con group was subcutaneously injected with saline daily for 21 consecutive days. For the Con group, the same injection volume was applied to the same hair removal area of dorsal skin. At 1 day post-bleomycin induction, the BLM+ATG group was intraperitoneally injected with $3 \mathrm{mg} / \mathrm{kg} /$ day ATG (Sigma-Aldrich; Merck KGaA) for 28 consecutive days $(10,15)$. ATG was dissolved into a stock solution at a concentration of $12 \mathrm{mg} / \mathrm{ml}$ using DMSO and diluted to a total concentration of $0.3 \mathrm{mg} / \mathrm{ml}$ with saline before administration. The Con and BLM group were intraperitoneally injected with saline (230 $\mu \mathrm{l}$ ) daily for 28 consecutive days. At the end of treatment, mice were anaesthetized with an intraperitoneal injection of pentobarbital sodium solution $(1 \% ; 40 \mathrm{mg} / \mathrm{kg})$ and sacrificed by cervical dislocation prior to collection of skin tissue samples. Then, skin tissue samples were collected from the defined area $\left(1 \mathrm{~cm}^{2}\right)$ injected with BLM. A schematic diagram of the experiment is presented in Fig. 1A.

Histologic examination. Hematoxylin and eosin (H\&E) staining of skin tissues was performed for pathological examination. Skin tissue samples were fixed in $4 \%$ paraformaldehyde at $4^{\circ} \mathrm{C}$ for $24 \mathrm{~h}$, embedded in paraffin and cut into 4- $\mu \mathrm{m}$ thick sections. The sections were stained with hematoxylin for $5 \mathrm{~min}$ and eosin for $3 \mathrm{~min}$ at room temperature. Calculation and analysis of dermal thickness were performed according to Sekiguchi et al (19). Briefly, dermal thickness was calculated as the distance from the epidermis-dermis junction to the dermis-subcutaneous tissue junction. Stained skin tissues were observed in six randomly selected fields of view using an Axio Scan.Z1 light microscope (magnification, x100; Carl Zeiss AG). ImageJ software (version 1.51, National Institutes of Health) was used to analyze dermal thickness.

Quantitative detection of collagen content. Collagen content in skin tissues was measured using a Sircol Collagen Assay kit (Biocolor, Ltd.) according to previous reports $(20,21)$ and the manufacturer's instructions. For analysis, skin was homogenized in $0.5 \mathrm{M}$ acetic acid containing $0.1 \mathrm{mg} / \mathrm{ml}$ pepsin (Sigma-Aldrich; Merck KGaA) with TissueLyser II LT (Qiagen, Inc.) at $4^{\circ} \mathrm{C}$, after which the supernatants were collected through centrifugation $\left(3,000 \times \mathrm{g}, 10 \mathrm{~min}, 4^{\circ} \mathrm{C}\right)$ and assayed.

Immunofluorescence staining. For immunofluorescence staining, full-thickness skin samples were dissected, attached to filter paper and fixed in $4 \%$ buffered paraformaldehyde overnight at $4^{\circ} \mathrm{C}$. The samples were embedded in optimal cutting temperature compound and prepared into $10-\mu \mathrm{m}$ thick sections using a CM3000 cryostat (Leica Microsystems $\mathrm{GmbH}$ ). After blocking for $30 \mathrm{~min}$ at room temperature with $10 \%$ goat serum (Sigma-Aldrich; Merck KGaA), the sections were incubated with anti-TGF- $\beta 1$ (1:100; Santa Cruz Biotechnology, Inc.; cat. no. sc146) and anti- $\alpha$-SMA (1:100; Abcam; cat. no. ab32575) primary antibodies overnight at $4^{\circ} \mathrm{C}$. After washing with $0.01 \mathrm{M}$ PBS three times, the sections were incubated with Alexa Fluor 488 conjugated anti rabbit secondary antibodies (1:1,000; Abcam; cat. no. ab150077) for 
A

$\begin{array}{ccc}\text { L } & 28 \text { days } \\ \begin{array}{ccc}\text { subcutaneous injection of } \\ \text { BLM or saline }\end{array} & \text { intraperitoneal injection of } & \text { 49 days } \\ \text { ATG or saline } & \text { Euthanasia }\end{array}$

B
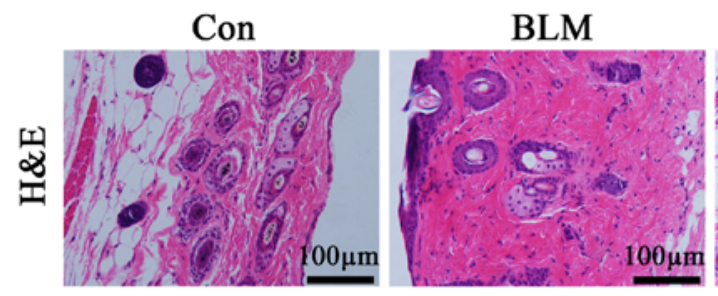

BLM+ATG

C

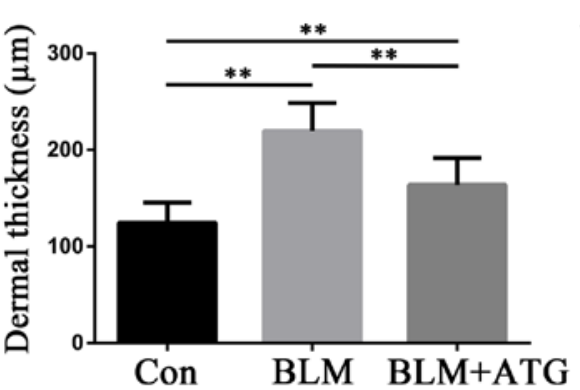

D

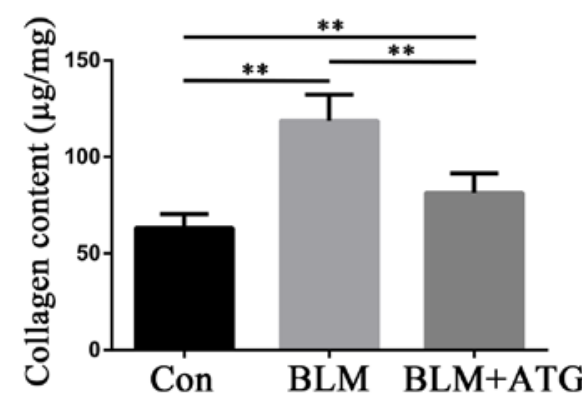

E

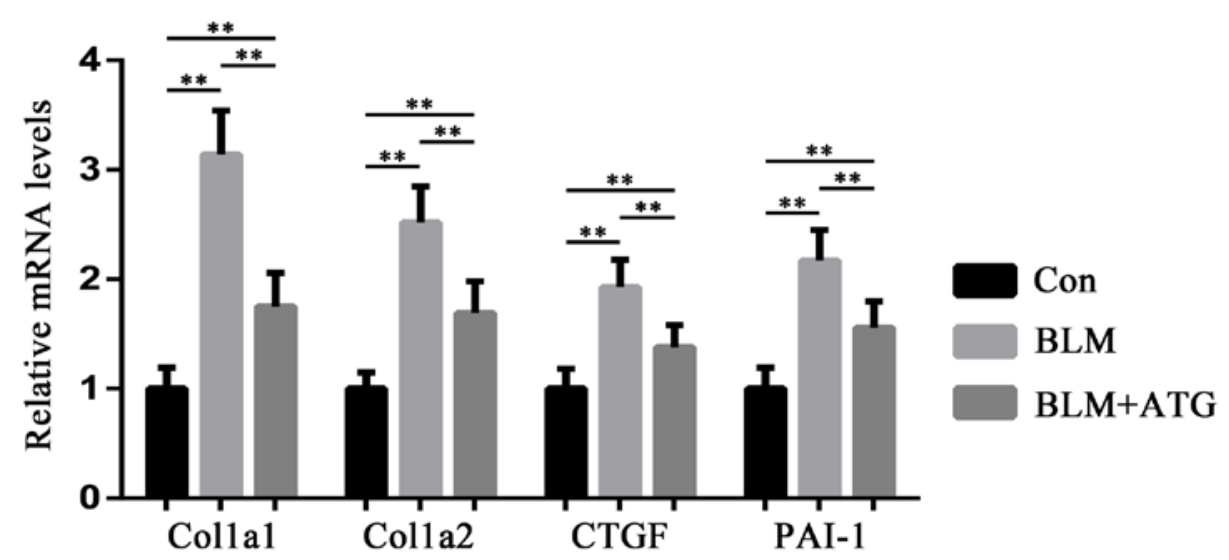

Figure 1. ATG alleviates BLM-induced dermal fibrosis in a murine HS model. (A) Schematic diagram of the experiment. (B) Representative images of H\&E staining of skin tissues. (C) Dermal thickness was calculated by measuring the distance from the epidermis-dermis junction to the dermis-subcutaneous tissue junction of H\&E stained sections. (D) Collagen content in the skin was detected using the Sircol collagen assay kit. (E) Extracellular matrix-related gene expression levels were measured via reverse transcription-quantitative PCR. ${ }^{* *} \mathrm{P}<0.01$. ATG, arctigenin; BLM, bleomycin; HS, hypertrophic scar; H\&E, Hematoxylin and eosin; Col1a1, collagen type I $\alpha 1$ chain; Colla2, collagen type I $\alpha 2$ chain; CTGF, connective tissue growth factor; PAI-1, plasminogen activator inhibitor-1; Con, Control.

$1 \mathrm{~h}$ at room temperature. Subsequently, cell nuclei were stained with DAPI (Sigma-Aldrich; Merck KGaA) for $10 \mathrm{~min}$ at room temperature. Stained sections were visualized using a TE2000 inverted fluorescence microscope (magnification, x200; Nikon Corporation) in at least six randomly selected fields of view.

Reverse transcription-quantitative $P C R$ (RT-qPCR). Total RNA was extracted from skin tissues using TRIzol ${ }^{\circledR}$ (Invitrogen; Thermo Fisher Scientific, Inc.). Total RNA was reverse transcribed into cDNA using the PrimeScript RT reagent kit (Takara Biotechnology Co., Ltd.). The temperature protocol for reverse transcription was: $37^{\circ} \mathrm{C}$ for $15 \mathrm{~min}$, followed by $85^{\circ} \mathrm{C}$ for $5 \mathrm{sec}$. Subsequently, qPCR was performed using SYBR Premix Ex Taq (Takara Biotechnology Co., Ltd.) and an ABI StepOne Plus Real-Time PCR machine
(Applied Biosystems; Thermo Fisher Scientific, Inc.). The following thermocycling conditions were used for qPCR: An initial denaturation at $95^{\circ} \mathrm{C}$ for $30 \mathrm{sec}$; followed by 40 cycles at $95^{\circ} \mathrm{C}$ for $5 \mathrm{sec}$ and $60^{\circ} \mathrm{C}$ for $30 \mathrm{sec}$; a final dissociation at $95^{\circ} \mathrm{C}$ for $60 \mathrm{sec}, 55^{\circ} \mathrm{C}$ for $30 \mathrm{sec}$ and $95^{\circ} \mathrm{C}$ for $60 \mathrm{sec}$. The primer sequences used for qPCR are presented in Table I. The $2^{-\triangle \Delta C q}$ method was used to calculate the relative gene expression levels (22).

Western blotting. Total proteins were extracted from skin tissues using RIPA (1\% PMSF) tissue lysate (Beyotime Institute of Biotechnology). Nuclear proteins were extracted using nuclear and cytoplasmic protein extraction kit (Beyotime Institute of Biotechnology). A bicinchoninic acid assay kit was used to measure the protein concentration. Proteins $(40 \mu \mathrm{g}$ 
Table I. Sequences of primers used for reverse transcriptionquantitative PCR.

\begin{tabular}{|c|c|}
\hline Gene & Sequence $\left(5^{\prime} \rightarrow 3^{\prime}\right)$ \\
\hline Colla1 & $\begin{array}{l}\text { F: GCTCCTCTTAGGGGCCACT } \\
\text { R: ATTGGGGACCCTTAGGCCAT }\end{array}$ \\
\hline Col1a2 & $\begin{array}{l}\text { F: TCGTGCCTAGCAACATGCC } \\
\text { R: TTTGTCAGAATACTGAGCAGCAA }\end{array}$ \\
\hline CTGF & $\begin{array}{l}\text { F: GGCCTCTTCTGCGATTTCG } \\
\text { R: GCAGCTTGACCCTTCTCGG }\end{array}$ \\
\hline PAI-1 & $\begin{array}{l}\text { F: GGCCTCTTCTGCGATTTCG } \\
\text { R: GCAGCTTGACCCTTCTCGG }\end{array}$ \\
\hline$\alpha-\mathrm{SMA}$ & $\begin{array}{l}\text { F: AGAAACGGGACAAACTTCGTC } \\
\text { R: GTCTTGCACTGTATAGCCGAG }\end{array}$ \\
\hline TGF- $\beta 1$ & $\begin{array}{l}\text { F: CCACCTGCAAGACCATCGAC } \\
\text { R: CTGGCGAGCCTTAGTTTGGAC }\end{array}$ \\
\hline IL-1 $\beta$ & $\begin{array}{l}\text { F: GAAATGCCACCTTTTGACAGTG } \\
\text { R: TGGATGCTCTCATCAGGACAG }\end{array}$ \\
\hline IL-4 & $\begin{array}{l}\text { F: CCCCAGCTAGTTGTCATCCTG } \\
\text { R: CAAGTGATTTTTGTCGCATCCG }\end{array}$ \\
\hline IL-6 & $\begin{array}{l}\text { F: CTGCAAGAGACTTCCATCCAG } \\
\text { R: AGTGGTATAGACAGGTCTGTTGG }\end{array}$ \\
\hline TNF- $\alpha$ & $\begin{array}{l}\text { F: CAGGCGGTGCCTATGTCTC } \\
\text { R: CGATCACCCCGAAGTTCAGTAG }\end{array}$ \\
\hline MCP-1 & $\begin{array}{l}\text { F: TAAAAACCTGGATCGGAACCAAA } \\
\text { R: GCATTAGCTTCAGATTTACGGGT }\end{array}$ \\
\hline GAPDH & $\begin{array}{l}\text { F: AGGTCGGTGTGAACGGATTTG } \\
\text { R: GGGGTCGTTGATGGCAACA }\end{array}$ \\
\hline
\end{tabular}

Colla1, collagen type I alpha 1 chain; Colla2, collagen type I alpha 2 chain; CTGF, connective tissue growth factor; PAI-1, plasminogen activator inhibitor-1; $\alpha$-SMA, $\alpha$-smooth muscle actin; MCP-1, monocyte chemoattractant protein-1; F, forward; R, reverse.

per lane) were separated via 10\% SDS-PAGE gels and transferred onto PVDF membranes (EMD Millipore). Then, the membranes were blocked for $2 \mathrm{~h}$ at room temperature using $5 \%$ skimmed milk powder. After blocking, the membranes were incubated overnight at $4^{\circ} \mathrm{C}$ with primary antibodies (all purchased from Abcam) targeted against: $\alpha$-SMA $(1: 1,000$; cat. no. ab5694), TGF- $\beta 1$ (1:1,000; cat. no. ab92486), nuclear factor erythroid-2-related factor 2 (Nrf2; nuclear; 1:1,000; cat. no. ab62352), heme oxygenase-1 (HO-1; 1:1,000; cat. no. ab13248) and GAPDH (1:2,000; cat. no. ab9485). After washing three times with TBS-T (TBS with $0.1 \%$ Tween-20) for $5 \mathrm{~min}$ each, the membranes were incubated with HRP-conjugated secondary antibodies (1:3,000; ProteinTech Group, Inc.; cat. no. SA00001-2) for $1 \mathrm{~h}$ at room temperature. Protein bands were visualized with ECL WB detection reagent (Beyotime Institute of Biotechnology) and imaged using the ChemiDoc ${ }^{\mathrm{TM}}$ XRS Imaging System (Bio-Rad Laboratories, Inc.). Protein expression levels were semi-quantified using ImageJ software (version 1.51, National Institutes of Health) with GAPDH as the loading control.
ELISA. The extracted skin tissues were weighed, minced, and homogenized using a TissueLyser II LT (Qiagen, Inc.) in $10 \mathrm{ml}$ cold phosphate-buffered saline, which included $1 \mathrm{mmol} / \mathrm{l}$ phenylmethylsulfonyl fluoride (Beyotime Institute of Biotechnology), $1 \%$ protease inhibitors cocktail (Sigma-Aldrich; Merck KGaA), 0.5\% sodium deoxycholate (Sigma-Aldrich; Merck KGaA) and 1\% Triton X-100 (Sigma-Aldrich; Merck KGaA). After incubation at $4^{\circ} \mathrm{C}$ for $1 \mathrm{~h}$, the homogenized mixtures were centrifuged at $10,000 \mathrm{xg}$ for $20 \mathrm{~min}$ at $4^{\circ} \mathrm{C}$. The supernatants were collected and stored at $-80^{\circ} \mathrm{C}$ prior to use. IL- $1 \beta$, IL- 4, IL- 6, TNF- $\alpha$, and monocyte chemoattractant protein-1 (MCP-1) contents in skin tissue homogenate supernatants were measured using ELISA kits (Jingmei Biotechnology; IL-1 $\beta$, cat. no. JM-02323M1; IL-4, cat. no. JM-02448M1; IL-6, cat. no. JM-02446M1; TNF- $\alpha$, cat. no. JM-02415M1; and MCP-1, cat. no. JM-02365M1) according to the manufacturer's protocol.

Determination of oxidative stress levels. Superoxide dismutase (SOD) activity, glutathione (GSH) concentration and malondialdehyde (MDA) levels in skin tissue homogenate supernatants, obtained by the aforementioned method, were measured spectrophotometrically at 450, 420 and $532 \mathrm{~nm}$ according to previous reports $(23,24)$ and the manufacturer's protocol (Nanjing Jiancheng Bioengineering Institute; SOD, cat. no. A001-3-2; GSH, cat. no. A006-1-1; MDA, cat. no. A003-1-2).

Statistical analysis. Statistical analyses were performed using SPSS software (version 21.0; IBM Corp.). Data are presented as the mean $\pm \mathrm{SD}$. Comparisons among multiple groups were analyzed using one-way ANOVA followed by the LSD post hoc test. $\mathrm{P}<0.05$ was considered to indicate a statistically significant difference. All experiments were repeated at least three times.

\section{Results}

ATG alleviates BLM-induced dermal fibrosis in a murine HS model. To evaluate the antifibrotic effect of ATG on HSs in vivo, BLM-induced skin fibrosis model mice were treated with ATG to examine its effects on dermal thickness, collagen content and extracellular matrix-related gene expression. H\&E staining suggested that BLM significantly induced dermal thickening compared with the Con group, whereas ATG significantly inhibited the fibrotic effect of BLM (Fig. 1B and C). Additionally, Sircol collagen detection also suggested that BLM-induced increases in collagen content were significantly suppressed by ATG treatment (Fig. 1D). In addition, Colla1, Col1a2, CTGF and PAI-1, which are important fibrotic components in the extracellular matrix, are overexpressed in HS (25). The RT-qPCR results indicated that the relative expression levels of the four aforementioned genes were significantly decreased in the BLM+ATG group compared with the BLM group (Fig. 1E). Collectively, the results indicated that ATG inhibited BLM-induced dermal fibrosis in vivo.

ATG inhibits the transformation of fibroblasts into myofibroblasts in vivo. By investigating the mechanism underlying 
A

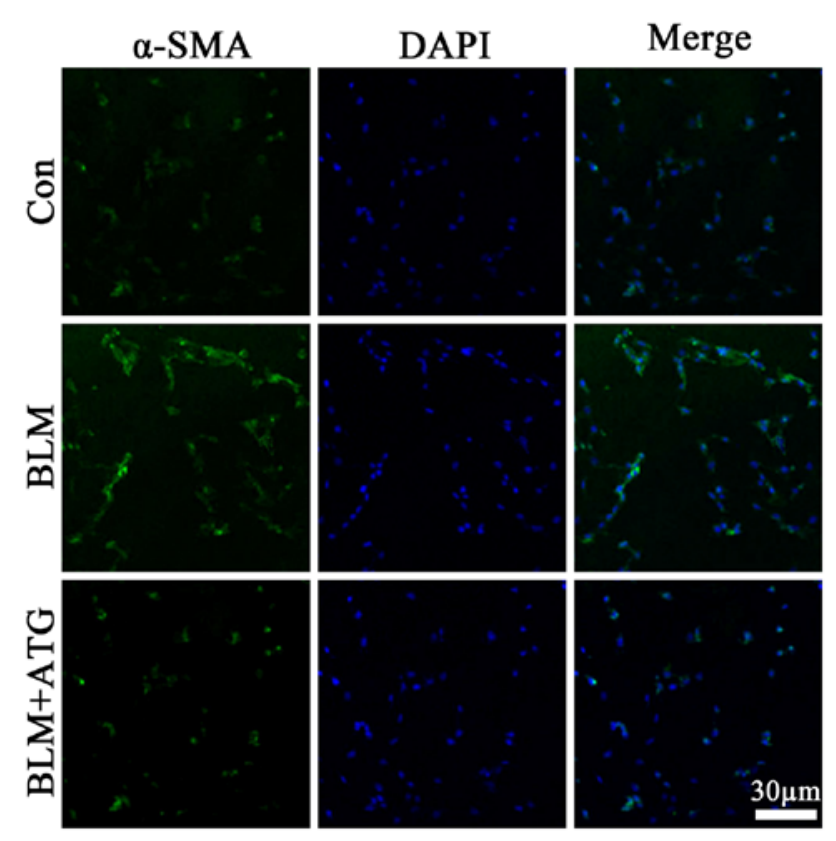

B
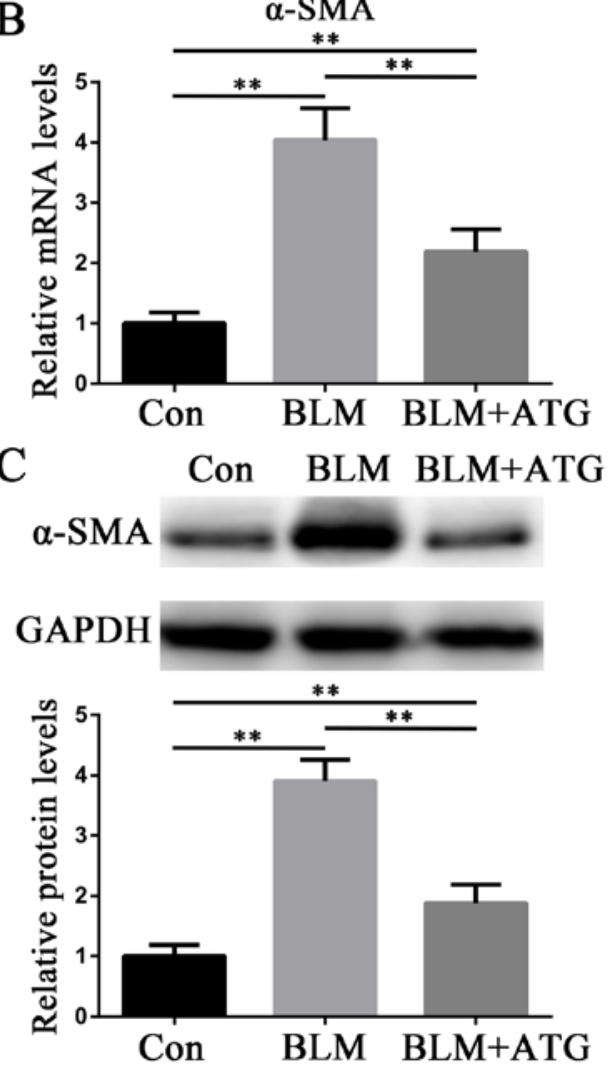

Figure 2. ATG inhibits the transformation of fibroblasts into myofibroblasts in vivo. (A) Representative fluorescence images of skin sections stained against $\alpha$-SMA (green) to identify myofibroblasts. Nuclei were counterstained with DAPI (blue). Scale bar, $30 \mu$ m; magnification, x200. (B) $\alpha$-SMA mRNA expression levels were measured via reverse transcription-quantitative PCR. (C) $\alpha$-SMA protein expression levels were measured via western blotting. ${ }^{* *} \mathrm{P}<0.01$. ATG, arctigenin; $\alpha$-SMA, $\alpha$-smooth muscle actin; BLM, bleomycin; Con, control.

HSs, it has been reported that myofibroblasts influence skin fibrosis (6). Myofibroblasts are primarily derived from activated fibroblasts and also have the characteristics of smooth muscle cells, with $\alpha$-SMA serving as hallmark (26). Therefore, $\alpha$-SMA has been demonstrated to serve as the primary biomarker for the phenotypic transformation of fibroblasts into myofibroblasts (26). In the present study, the expression level of $\alpha$-SMA in skin tissues was detected via immunofluorescence staining (Fig. 2A), RT-qPCR (Fig. 2B) and western blotting (Fig. 2C). The expression level of $\alpha$-SMA was markedly upregulated in the BLM group compared with the Con group, whereas $\alpha$-SMA expression levels were notably downregulated in the BLM+ATG group compared with the BLM group (Fig. 2A-C). The results indicated that ATG inhibited the differentiation of fibroblasts into myofibroblasts in vivo.

ATG downregulates the expression of TGF- $\beta 1$ in BLMinduced skin fibrosis model mice. TGF- $\beta 1$, a profibrotic growth factor, serves as the primary effector molecule in skin fibrosis (27). Immunofluorescence staining (Fig. 3A), RT-qPCR (Fig. 3B) and western blotting (Fig. 3C) were performed to analyze the expression of TGF- $\beta 1$. Compared with the Con group, BLM markedly increased TGF- $\beta 1$ expression levels, which were reversed by ATG treatment (Fig. 3A-C). The results suggested that ATG could reverse elevated TGF- $\beta 1$ expression levels in BLM-induced skin fibrosis model mice.
ATG reduces the inflammatory response in BLM-induced skin fibrosis model mice. Increasing evidence has demonstrated that inflammation is a key mechanism underlying the initiation and maintenance of pathological fibrosis, including HSs $(28,29)$. Multiple cytokines, such as IL-1 $\beta$, IL-4, IL-6, TNF- $\alpha$ and MCP-1, serve a central role in orchestrating chronic inflammation (30). To study the anti-inflammatory effects of ATG, the expression levels of the aforementioned inflammatory factors were measured. The RT-qPCR results indicated that the mRNA expression levels of IL-1 $\beta$, IL-4, IL-6, TNF- $\alpha$ and MCP-1 were significantly increased in the BLM group compared with the Con group, but ATG treatment significantly inhibited BLM-induced mRNA expression levels (Fig. 4A). ELISAs were also conducted to further investigate the aforementioned results (Fig. 4B). The ELISA results were consistent with those of RT-qPCR, indicating that reducing the inflammatory response may serve as a mechanism underlying ATG-mediated amelioration of skin fibrosis.

ATG reduces oxidative stress in BLM-induced skin fibrosis model mice. Previous studies have demonstrated that elevated oxidative stress and redox imbalance exacerbate the pathogenesis of skin fibrosis $(31,32)$. Therefore, identifying the effect of ATG treatment in modulating oxidative stress requires investigation. The present study indicated that the BLM+ATG group displayed significantly increased SOD activity and GSH 
A

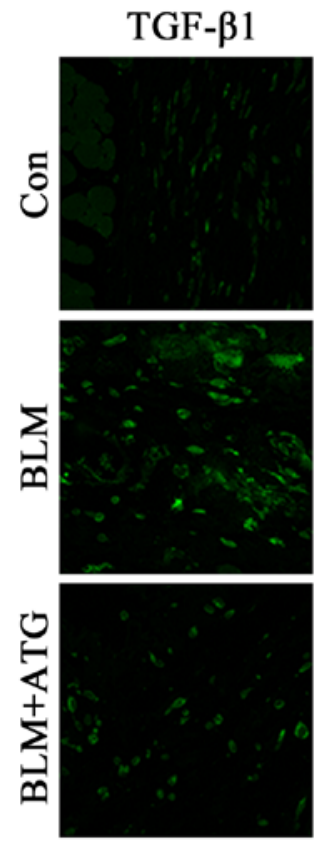

DAPI
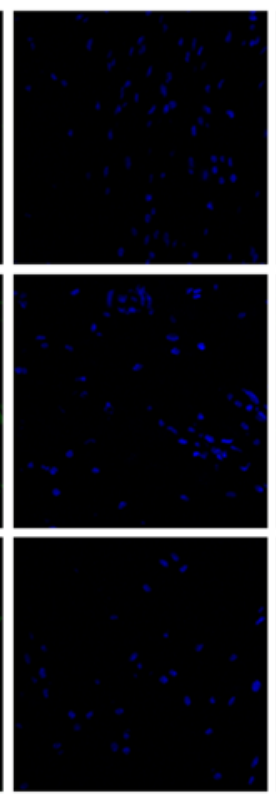

Merge

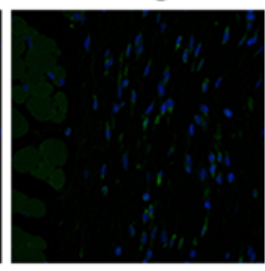

$=\mathrm{C}$
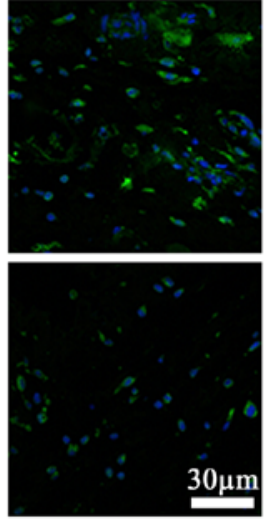

B

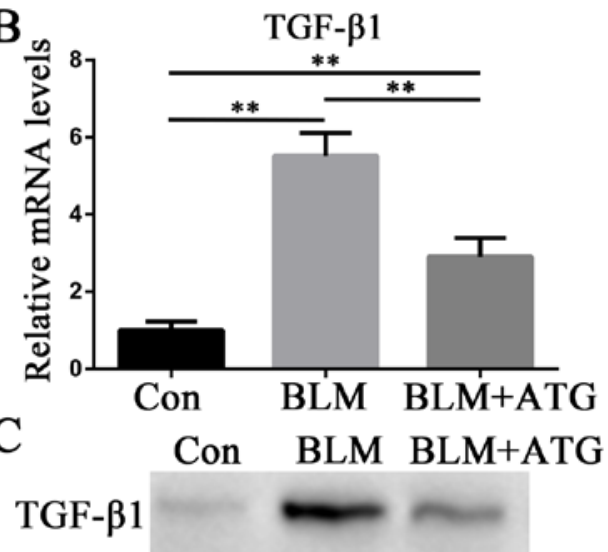

GAPDH

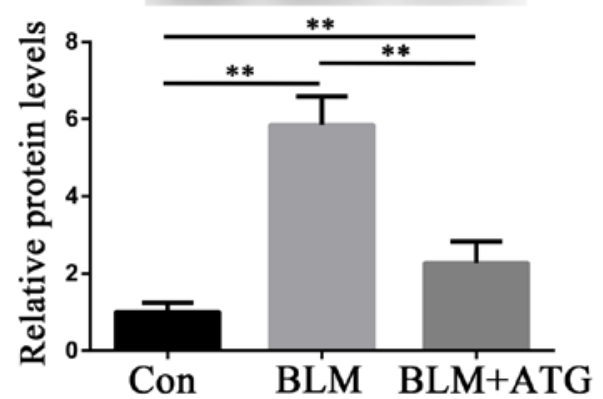

Figure 3. ATG downregulates the expression of TGF- $\beta 1$ in BLM-induced skin fibrosis model mice. (A) Representative fluorescence images of skin sections stained against TGF- $\beta 1$ (green). Nuclei were counterstained with DAPI (blue). Scale bar, $30 \mu$ m; magnification, x200. (B) TGF- $\beta 1$ mRNA expression levels were measured via reverse transcription-quantitative PCR. (C) TGF- $\beta 1$ protein expression levels were measured via western blotting. ${ }^{* *} \mathrm{P}<0.01$. ATG, arctigenin; BLM, bleomycin; Con, control.

A

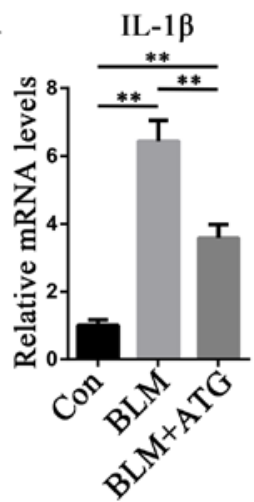

B

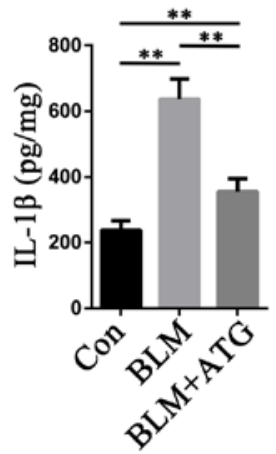

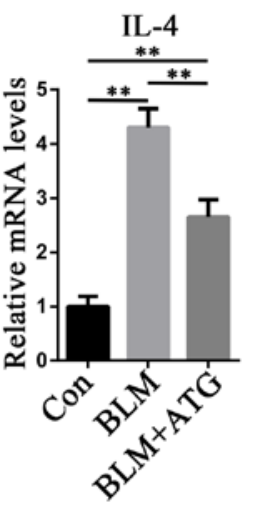

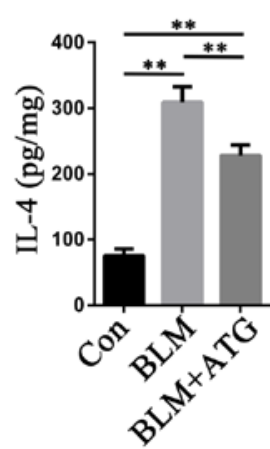

IL-6
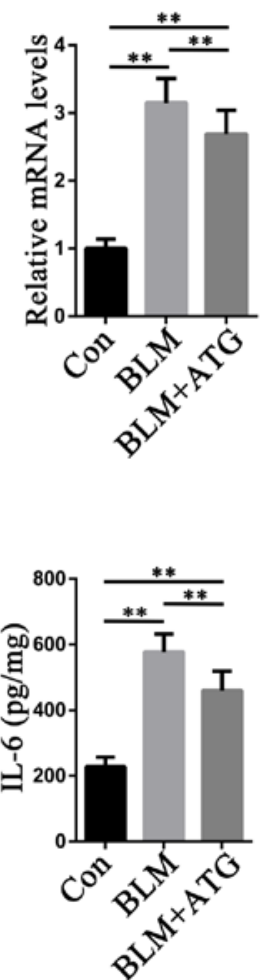

TNF- $\alpha$
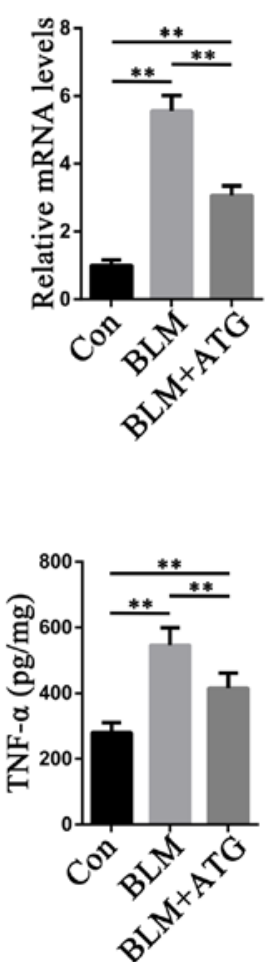

MCP-1
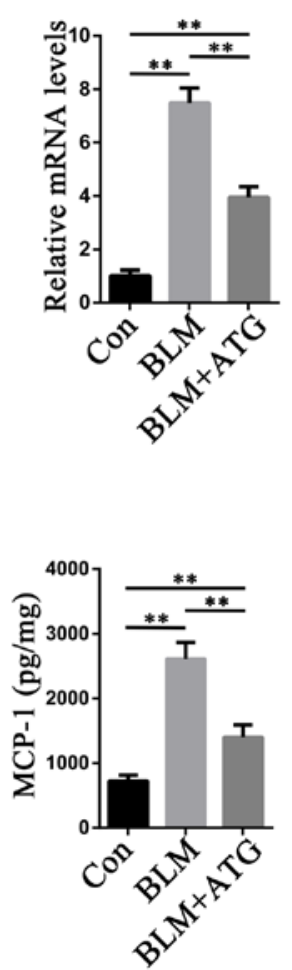

Figure 4. ATG reduces the inflammation response in BLM-induced skin fibrosis model mice. (A) mRNA expression levels of IL-1 $\beta$, IL-4, IL-6, TNF- $\alpha$ and MCP-1 were measured via reverse transcription-quantitative PCR. (B) Protein expression levels of IL- $1 \beta$, IL- 4 , IL- 6 , TNF- $\alpha$ and MCP-1 were measured using ELISAs in skin tissue homogenate supernatants. ${ }^{* *} \mathrm{P}<0.01$. ATG, arctigenin; BLM, bleomycin; MCP-1, monocyte chemoattractant protein-1; Con, control. 

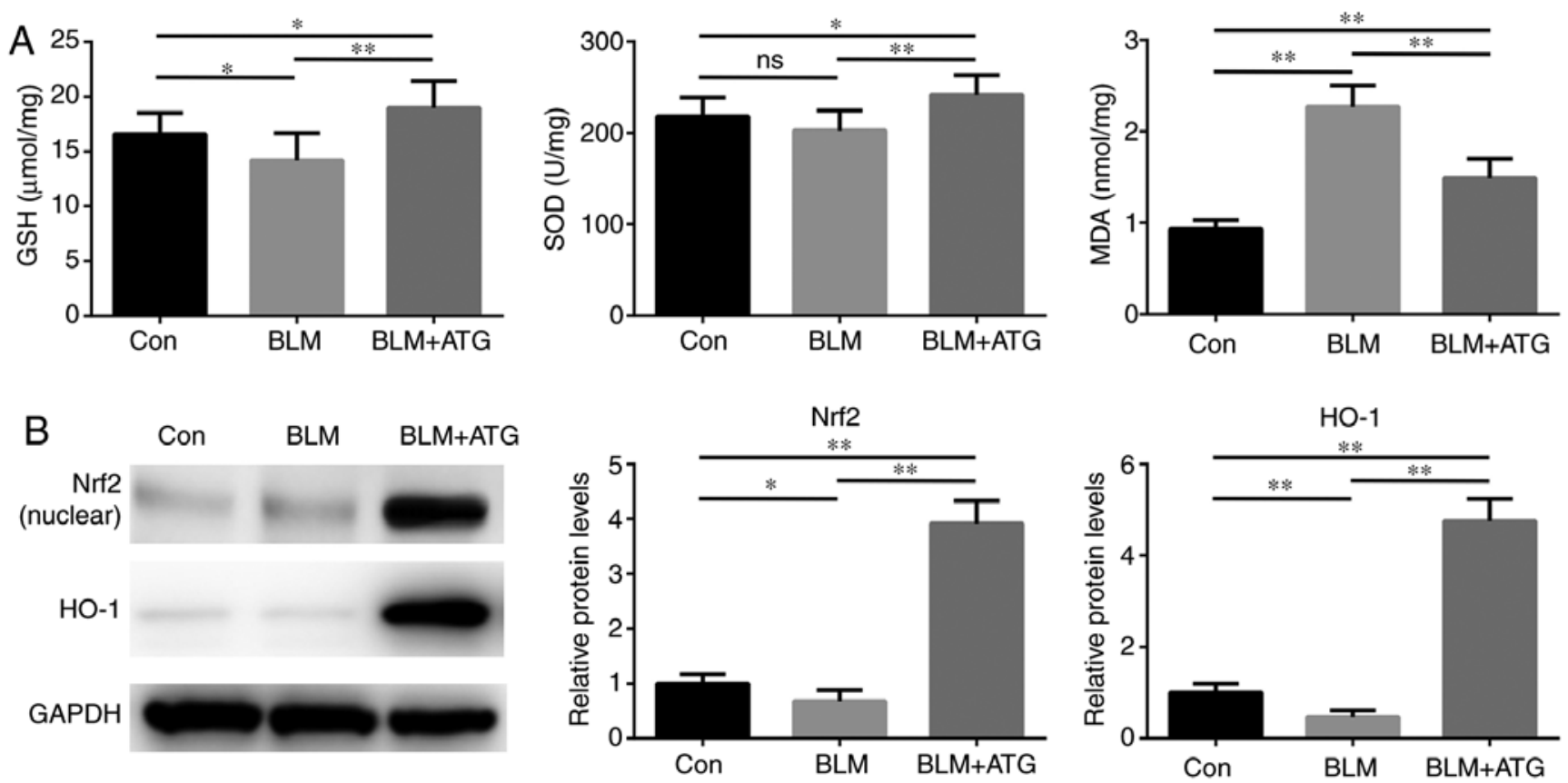

Figure 5. ATG reduces oxidative stress in BLM-induced skin fibrosis model mice. (A) The levels of GSH, SOD and MDA were detected spectrophotometrically in skin tissue homogenate supernatants. (B) Nrf2 (nuclear) and HO-1 protein expression levels were measured via western blotting. ${ }^{*}<0.05$ and ${ }^{* * *} \mathrm{P}<0.01$. ATG, arctigenin; BLM, bleomycin; GSH, glutathione; SOD, superoxide dismutase; MDA, malondialdehyde; Nrf2, nuclear factor erythroid-2-related factor 2; HO-1, heme oxygenase-1; Con, control.

concentration, but significantly decreased MDA levels compared with the BLM group, which may be attributed to the antioxidant effect of ATG (Fig. 5A).

To further investigate the antioxidant mechanism underlying ATG, the expression of components of the $\mathrm{Nrf} / \mathrm{HO}-1$ signaling pathway, which serves a critical negative regulatory role in oxidative stress, was assessed $(33,34)$. BLM significantly downregulated the protein expression levels of Nrf2 (nuclear) and HO-1 compared with the Con group (Fig. 5B). Nrf2 and HO-1 expression levels were significantly increased in the BLM+ATG group compared with the BLM group. Collectively, the results indicated that ATG effectively resolved skin fibrosis by reducing oxidative stress via activation of the Nrf2/HO-1 signaling pathway.

\section{Discussion}

HSs are a progressive fibroproliferation disease characterized by the continuous activation of fibroblasts and excessive deposition of extracellular matrix (1). At present, no effective treatment strategy for HS has been established, which is primarily attributed to the complicated pathological processes mediated by a series of underlying mechanisms (5). A variety of biologically active compounds derived from natural products have been developed into drugs due to their potential pharmacological activities and reduced side effects $(35,36)$. Among these, ATG has received increasing attention due to its reported antifibrotic potential in several diseases, including oral submucous fibrosis, peritoneal fibrosis, obstructive nephropathy and liver fibrosis (13-17). However, the antifibrotic effects of ATG on HSs are not completely understood. To the best of our knowledge, the present study was the first to provide evidence for ATG as a potential therapeutic agent against skin fibrosis in HS via reducing inflammation and oxidative stress.

In the present study, the inhibitory effect of ATG on skin fibrosis was investigated by performing histological staining and collagen content determination. Subsequently, the expression of extracellular matrix-related genes in the skin, including Colla1, Col1a2, CTGF and PAI-1, was measured. Colla1 and Colla2 are two genes involved in the biosynthesis of type 1 collagen (37), CTGF is a key factor in maintaining profibrotic milieu (38) and PAI-1 functions as a suppressor of fibrinolysis (39). The results of the present study indicated that Col1a1, Col1a2, CTGF and PAI-1 expression levels were decreased in the BLM+ATG group compared with the BLM group. The excessive accumulation of extracellular matrix is attributable to the pathological recruitment of fibroblasts to injured sites and their transformation to $\alpha$-SMA-expressing myofibroblasts $(6,26)$. Although the regulatory mechanisms underlying fibroblast activation are not completely understood, numerous studies have demonstrated that TGF- $\beta 1$ serves as the main effector (40-42). The present study indicated that ATG treatment significantly decreased the expression of $\alpha$-SMA and TGF- $\beta 1$ in BLM-induced fibrotic skin, suggesting that the antifibrotic effect of ATG was associated with suppression of TGF- $\beta 1$-mediated ECM gene transcription and fibroblast activation.

Despite extensive efforts to elucidate the possible mechanisms underlying fibrosis, the understanding of the initiation and progression of fibrosis is limited. A number of studies have demonstrated that inflammation is essential in modulating several pathological processes of skin fibrosis $(28,29)$. In the early stage, immune cells are activated by various stimuli, which leads to the secretion of inflammatory factors that induce the expression of adhesion molecules on the surface of endothelial cells. Endothelial cell-specific adhesion 
molecules interact with immune cells, recruiting the cells to sites of injury. Therefore, chronic inflammation may be evoked and sustained by a positive feedback loop (43). It has been previously reported that the levels of inflammatory factors are higher in fibrotic skin compared with healthy skin (29). The results of the present study supported the aforementioned finding. Moreover, the results indicated that ATG treatment decreased BLM-induced upregulation of mRNA expression levels of several inflammatory factors. Collectively, the results suggested that the antifibrotic effects of ATG might be related to its anti-inflammatory effects.

Recent studies have demonstrated that oxidative stress is enhanced in BLM-induced skin fibrosis, which has been hypothesized to be associated with fibroblast activation and the generation of inflammatory factors $(23,44,45)$. MDA is a product of reactive species-induced lipid peroxidation and is an indicator of oxidative stress (46). In addition, GSH and SOD, which are intracellular antioxidant enzymes, can be consumed by free radicals (47). The results of the present study indicated that ATG reduced oxidative stress in BLM-induced fibrotic skin, as demonstrated by the upregulation of antioxidants (GSH and SOD) and downregulation of oxidants (MDA) in the BLM+ATG group compared with the BLM group. It has been reported that activation of the Nrf2/HO-1 signaling pathway serves a pivotal role in antioxidative defense $(33,34)$. As a redox-sensitive transcription factor, activated Nrf2 can translocate from the cytoplasm into the nucleus and bind to antioxidant response elements (AREs) on the HO-1 promoter (48). In the present study, compared with the BLM group, ATG promoted nuclear translocation of $\mathrm{Nrf} 2$ and increased the expression of HO-1 in BLM-induced fibrotic skin, suggesting that the antioxidant mechanism underlying ATG might be associated with activation of the Nrf2-mediated signaling pathway.

The present study primarily focused on the antifibrotic effects of ATG on HSs without considering the route of administration. In the present study, ATG was administered via intraperitoneal injection, which had the advantage of avoiding the first-pass metabolism effect and improving bioavailability as a parenteral administration method. However, intraperitoneal administration is not a suitable for use in the clinic. Zhong et al (9) recently reported that oral gavage is also a suitable application route for ATG, which had been shown to exert a protective effect in diabetic kidney disease. In addition, it was hypothesized that if ATG could be formulated into a plaster and applied to the skin surface, it may display an improved therapeutic effect; however, low transdermal ability remains a challenge. In addition, the timing and dose of administration require further investigation. Moreover, the animal model of BLM-induced skin fibrosis used in the present study did not cause skin ulceration, thus, the healing time could not be assessed. Future studies should establish an animal model of skin resection to evaluate the effect of ATG on healing time.

In summary, the present study indicated that ATG ameliorated skin fibrosis in a murine HS model. The antifibrotic effects of ATG may be mediated in part by reducing inflammation and oxidative stress. Therefore, the present study suggested that ATG may serve as a therapeutic agent for HSs.

\section{Acknowledgements}

Not applicable.

\section{Funding}

No funding was received.

\section{Availability of data and materials}

The datasets used and/or analyzed during the current study are available from the corresponding author on reasonable request.

\section{Authors' contributions}

LJ and YL contributed to the study design. LJ, YD, WL and YL performed the experiments and analyzed the data. LJ wrote the manuscript. YD, WL and YL edited the manuscript. All authors read and approved the final manuscript.

\section{Ethics approval and consent to participate}

The present study was approved by the Ethics Committee of Chongqing University Central Hospital (approval no. 2018-032).

\section{Patient consent for publication}

Not applicable.

\section{Competing interests}

The authors declare that they have no competing interests.

\section{References}

1. Zhang J, Li Y, Bai X, Li Y, Shi J and Hu D: Recent advances in hypertrophic scar. Histol Histopathol 33: 27-39, 2018.

2. Niessen FB, Spauwen PH, Schalkwijk J and Kon M: On the nature of hypertrophic scars and keloids: A review. Plast Reconstr Surg 104: 1435-1458, 1999.

3. Tyack Z, Simons M, Spinks A and Wasiak J: A systematic review of the quality of burn scar rating scales for clinical and research use. Burns 38: 6-18, 2012

4. Brown BC, McKenna SP, Siddhi K, McGrouther DA and Bayat A: The hidden cost of skin scars: Quality of life after skin scarring. J Plast Reconstr Aesthet Surg 61: 1049-1058, 2008.

5. Bloemen MC, van der Veer WM, Ulrich MM, van Zuijlen PP, Niessen FB and Middelkoop E: Prevention and curative management of hypertrophic scar formation. Burns 35: 463-475, 2009.

6. Jinnin M: Mechanisms of skin fibrosis in systemic sclerosis. J Dermatol 37: 11-25, 2010.

7. Cho MK, Jang YP, Kim YC and Kim SG: Arctigenin, a phenylpropanoid dibenzylbutyrolactone lignan, inhibits MAP kinases and AP-1 activation via potent MKK inhibition: The role in TNF-alpha inhibition. Int Immunopharmacol 4: 1419-1429, 2004.

8. Gao Q, Yang M and Zuo Z: Overview of the anti-inflammatory effects, pharmacokinetic properties and clinical efficacies of arctigenin and arctiin from Arctium lappa L. Acta Pharmacol Sin 39: 787-801, 2018

9. Zhong Y, Lee K, Deng Y, Ma Y, Chen Y, Li X, Wei C, Yang S, Wang T, Wong NJ, et al: Arctigenin attenuates diabetic kidney disease through the activation of PP2A in podocytes. Nat Commun 10: 4523, 2019.

10. Zhu Z, Yan J, Jiang W, Yao XG, Chen J, Chen L, Li C, Hu L, Jiang H and Shen X: Arctigenin effectively ameliorates memory impairment in Alzheimer's disease model mice targeting both $\beta$-amyloid production and clearance. J Neurosci 33: 13138-13149, 2013. 
11. Lee JY and Kim CJ: Arctigenin, a phenylpropanoid dibenzylbutyrolactone lignan, inhibits type I-IV allergic inflammation and pro-inflammatory enzymes. Arch Pharm Res 33: 947-957, 2010.

12. Lee JY, Cho BJ, Park TW, Park BE, Kim SJ, Sim SS and Kim CJ: Dibenzylbutyrolactone lignans from Forsythia koreana fruits attenuate lipopolysaccharide-induced inducible nitric oxide synthetase and cyclooxygenase- 2 expressions through activation of nuclear factor- $\kappa b$ and mitogen-activated protein kinase in RAW264.7 cells. Biol Pharm Bull 33: 1847-1853, 2010.

13. Lin CY, Hsieh PL, Liao YW, Peng CY, Yu CC and Lu MY: Arctigenin reduces myofibroblast activities in oral submucous fibrosis by LINC00974 inhibition. Int J Mol Sci 20: 1328, 2019.

14. Jin G, Su Y, Dong Q, Zhao X, Zhang L and Yan X: Arctigenin alleviates TGF- $\beta 1$-induced epithelial-mesenchymal transition and PAI-1 expression via AMPK/NF- $\kappa \mathrm{B}$ pathway in peritoneal mesothelial cells. Biochem Biophys Res Commun 520: 413-419, 2019.

15. Li A, Zhang X, Shu M, Wu M, Wang J, Zhang J, Wang R, Li P and Wang Y: Arctigenin suppresses renal interstitial fibrosis in a rat model of obstructive nephropathy. Phytomedicine 30: 28-41, 2017.

16. Li A, Wang J, Zhu D, Zhang X, Pan R and Wang R: Arctigenin suppresses transforming growth factor- $\beta 1$-induced expression of monocyte chemoattractant protein- 1 and the subsequent epithelial-mesenchymal transition through reactive oxygen species-dependent ERK/NF- $\kappa \mathrm{B}$ signaling pathway in renal tubular epithelial cells. Free Radic Res 49: 1095-1113, 2015.

17. Li A, Wang J, Wu M, Zhang X and Zhang $\mathrm{H}$ : The inhibition of activated hepatic stellate cells proliferation by arctigenin through G0/G1 phase cell cycle arrest: Persistent p27(Kip1) induction by interfering with PI3K/Akt/FOXO3a signaling pathway. Eur J Pharmacol 747: 71-87, 2015.

18. Dudgeon C, Shreeram S, Tanoue K, Mazur SJ, Sayadi A, Robinson RC, Appella E and Bulavin DV: Genetic variants and mutations of PPM1D control the response to DNA damage. Cell Cycle 12: 2656-2664, 2013.

19. Sekiguchi A, Motegi SI, Fujiwara C, Yamazaki S, Inoue Y, Uchiyama A, Akai R, Iwawaki T and Ishikawa O: Inhibitory effect of kaempferol on skin fibrosis in systemic sclerosis by the suppression of oxidative stress. J Dermatol Sci 96: 8-17, 2019.

20. Fujiwara C, Uehara A, Sekiguchi A, Uchiyama A, Yamazaki S, Ogino S, Yokoyama Y, Torii R, Hosoi M, Suto C, et al: Suppressive regulation by MFG-E8 of latent transforming growth factor $\beta$-induced fibrosis via binding to $\alpha \mathrm{v}$ integrin Significance in the pathogenesis of fibrosis in systemic sclerosis. Arthritis Rheumatol 71: 302-314, 2019.

21. Thoua NM, Derrett-Smith EC, Khan K, Dooley A, Shi-Wen X and Denton CP: Gut fibrosis with altered colonic contractility in a mouse model of scleroderma. Rheumatology (Oxford) 51 : 1989-1998, 2012

22. Livak KJ and Schmittgen TD: Analysis of relative gene expression data using real-time quantitative PCR and the 2(-Delta Delta $\mathrm{C}(\mathrm{T})$ ) method. Methods 25: 402-408, 2001

23. Zhou CF, Yu JF, Zhang JX, Jiang T, Xu SH, Yu QY and Zhu QX $\mathrm{N}$-acetylcysteine attenuates subcutaneous administration of bleomycin-induced skin fibrosis and oxidative stress in a mouse model of scleroderma. Clin Exp Dermatol 38: 403-409, 2013.

24. Umasuthan N, Bathige SD, Revathy KS, Lee Y, Whang I, Choi CY, Park HC and Lee J: A manganese superoxide dismutase (MnSOD) from Ruditapes philippinarum: Comparative structural- and expressional-analysis with copper/zinc superoxide dismutase (Cu/ZnSOD) and biochemical analysis of its antioxidant activities. Fish Shellfish Immunol 33: 753-765, 2012.

25. Liu Q, Lu J, Lin J, Tang Y, Pu W, Shi X, Jiang S, Liu J, Ma Y, Li Y,et al: Salvianolic acid B attenuates experimental skin fibrosis of systemic sclerosis. Biomed Pharmacother 110: 546-553, 2019.

26. Ju W, Zhihong Y, Zhiyou Z, Qin H, Dingding W, Li S, Baowei Z, Xing W, Ying $\mathrm{H}$ and An $\mathrm{H}$ : Inhibition of alpha-SMA by the ectodomain of FGFR2c attenuates lung fibrosis. Mol Med 18 992-1002, 2012.

27. Verrecchia F, Mauviel A and Farge D: Transforming growth factor-beta signaling through the Smad proteins: Role in systemic sclerosis. Autoimmun Rev 5: 563-569, 2006.

28. Zhang J, Qiao Q, Liu M, He T, Shi J, Bai X, Zhang Y, Li Y, Cai W, Han S, et al: IL-17 promotes scar formation by inducing macrophage infiltration. Am J Pathol 188: 1693-1702, 2018.
29. Shan S, Zhang Y, Wu M, Yi B, Wang J and Li Q: Naringenin attenuates fibroblast activation and inflammatory response in a mechanical stretch-induced hypertrophic scar mouse model. Mol Med Rep 16: 4643-4649, 2017.

30. Christmann RB and Lafyatis R: The cytokine language of monocytes and macrophages in systemic sclerosis. Arthritis Res Ther 12: 146, 2010.

31. Vona R, Giovannetti A, Gambardella L, Malorni W, Pietraforte D and Straface E: Oxidative stress in the pathogenesis of systemic scleroderma: An overview. J Cell Mol Med 22: 3308-3314, 2018.

32. Shroff A, Mamalis A and Jagdeo J: Oxidative stress and skin fibrosis. Curr Pathobiol Rep 2: 257-267, 2014.

33. Wu W, Peng G, Yang F, Zhang Y, Mu Z and Han X: Sulforaphane has a therapeutic effect in an atopic dermatitis murine model and activates the Nrf2/HO1 axis. Mol Med Rep 20: 1761-1771, 2019.

34. Kavian N, Mehlal S, Jeljeli M, Saidu NEB, Nicco C, Cerles O, Chouzenoux S, Cauvet A, Camus C, Ait-Djoudi M, et al: The Nrf2-antioxidant response element signaling pathway controls fibrosis and autoimmunity in scleroderma. Front Immunol 9: $1896,2018$.

35. Katz L and Baltz RH: Natural product discovery: Past, present, and future. J Ind Microbiol Biotechnol 43: 155-176, 2016.

36. Atanasov AG, Waltenberger B, Pferschy-Wenzig EM, Linder T, Wawrosch C, Uhrin P, Temml V, Wang L, Schwaiger S, Heiss EH, et al: Discovery and resupply of pharmacologically active plant-derived natural products: A review. Biotechnol Adv 33: 1582-1614, 2015.

37. Won YD, Na MK, Kim CH, Kim JM, Cheong JH, Ryu JI and Han MH: The frontal skull Hounsfield unit value can predict ventricular enlargement in patients with subarachnoid haemorrhage. Sci Rep 8: 10178, 2018.

38. Liu S, Taghavi R and Leask A: Connective tissue growth factor is induced in bleomycin-induced skin scleroderma. J Cell Commun Signal 4: 25-30, 2010.

39. Chung EJ, McKay-Corkum G, Chung S, White A, Scroggins BT, Mitchell JB, Mulligan-Kehoe MJ and Citrin D: Truncated plasminogen activator inhibitor-1 protein protects from pulmonary fibrosis mediated by irradiation in a murine model. Int J Radiat Oncol Biol Phys 94: 1163-1172, 2016.

40. Lichtman MK, Otero-Vinas $M$ and Falanga V: Transforming growth factor beta (TGF- $\beta$ ) isoforms in wound healing and fibrosis. Wound Repair Regen 24: 215-222, 2016.

41. Selvarajah B,Azuelos I,Platé M, Guillotin D,Forty EJ,Contento G, Woodcock HV, Redding M, Taylor A, Brunori G, et al: mTORC1 amplifies the ATF4-dependent de novo serine-glycine pathway to supply glycine during TGF- $\beta_{1}$-induced collagen biosynthesis. Sci Signal 12: eaav3048, 2019.

42. Lima JDCC, Simoes E, de Castro G, Morais MRPT, de Matos-Neto EM, Alves MJ, Pinto NI, Figueredo RG, Zorn TMT, Felipe-Silva AS, et al: Tumour-derived transforming growth factor- $\beta$ signalling contributes to fibrosis in patients with cancer cachexia. J Cachexia Sarcopenia Muscle 10: 1045-1059, 2019.

43. Zhong L, Simard MJ and Huot J: Endothelial microRNAs regulating the NF- $\kappa \mathrm{B}$ pathway and cell adhesion molecules during inflammation. FASEB J 32: 4070-4084, 2018

44. Doridot L, Jeljeli M, Chêne C and Batteux F: Implication of oxidative stress in the pathogenesis of systemic sclerosis via inflammation, autoimmunity and fibrosis. Redox Biol 25: 101122, 2019.

45. Zhou CF, Zhou DC, Zhang JX, Wang F, Cha WS, Wu CH and Zhu QX: Bleomycin-induced epithelial-mesenchymal transition in sclerotic skin of mice: Possible role of oxidative stress in the pathogenesis. Toxicol Appl Pharmacol 277: 250-258, 2014.

46. Czerska M, Mikolajewska K, Zieliński M, Gromadzińska J and Wąsowicz W: Today's oxidative stress markers. Med Pr 66: 393-405, 2015

47. Yang HY and Lee TH: Antioxidant enzymes as redox-based biomarkers: A brief review. BMB Rep 48: 200-208, 2015.

48. Liu Z, Wang J, Huang E, Gao S, Li H, Lu J, Tian K, Little PJ, Shen X, Xu S and Liu P: Tanshinone IIA suppresses cholesterol accumulation in human macrophages: Role of heme oxygenase-1. J Lipid Res 55: 201-213, 2014.

This work is licensed under a Creative Commons Attribution-NonCommercial-NoDerivatives 4.0 International (CC BY-NC-ND 4.0) License. 